\title{
Involved-Field Radiation Therapy for Locoregionally Recurrent Ovarian Cancer
}

\author{
Aaron P. Brown, M.D. ${ }^{a}$, Anuja Jhingran, M.D. ${ }^{a}$, Ann H. Klopp, M.D., Ph.D. ${ }^{a}$, Kathleen M. \\ Schmeler, M.D. ${ }^{b}$, Pedro T. Ramirez, M.D. ${ }^{b}$, and Patricia J. Eifel, M.D. ${ }^{a}$ \\ aDepartments of Radiation Oncology, The University of Texas MD Anderson Cancer Center, \\ Houston, TX, U.S.A \\ bDepartments of Gynecologic Oncology, The University of Texas MD Anderson Cancer Center, \\ Houston, TX, U.S.A
}

\section{Abstract}

Objective-To evaluate the effectiveness of definitive involved-field radiation therapy (IFRT) for selected patients with locoregionally-recurrent ovarian cancer.

\begin{abstract}
Methods-We retrospectively reviewed records of 102 epithelial ovarian cancer patients treated with definitive IFRT ( $\geq 45$ Gy). IFRT was directed to localized nodal (49\%) and extranodal (51\%) recurrences.
\end{abstract}

Results-The median time from diagnosis to IFRT was 36 months (range, 1-311), and the median follow-up after IFRT was 37 months (range, 1-123). Patients received a median of three chemotherapy courses before IFRT (range, 0-9). Five-year overall (OS) and progression-free survival (PFS) rates after IFRT were $40 \%$ and $24 \%$ respectively; the 5-year in-field disease control rate was $71 \%$.

Thirty-five patients (35\%) had no evidence of disease at a median of 38 months after IFRT (range, $7-122$ ), including 25 continuously without disease for a median of 61 months (range, 17-122) and 10 with salvage treatment following disease recurrence, disease-free for a median of 39 months after salvage treatment (range, 7-92).

Eight clear cell carcinoma patients had higher 5-year OS (88\% versus 37\%; $\mathrm{p}=0.05)$ and PFS ( $75 \%$ versus $20 \% ; \mathrm{p}=0.01$ ) rates than other patients. Patients sensitive to initial platinum chemotherapy had a higher 5-year OS rate than platinum-resistant patients (43\% versus $27 \%$,

() 2013 Elsevier Inc. All rights reserved.

Corresponding Author: Patricia J. Eifel, M.D., Department of Radiation Oncology, Unit 1202, Mays Clinic (ACBP1.2867), MD Anderson Cancer Center, 1515 Holcombe Blvd., Houston, TX 77030. Tel: 713-563-2343; Fax: 713-563-6940; peifel@mdanderson.org.

Publisher's Disclaimer: This is a PDF file of an unedited manuscript that has been accepted for publication. As a service to our customers we are providing this early version of the manuscript. The manuscript will undergo copyediting, typesetting, and review of the resulting proof before it is published in its final citable form. Please note that during the production process errors may be discovered which could affect the content, and all legal disclaimers that apply to the journal pertain.

Presented in part at the ASTRO 2012 annual meeting, Boston, MA, October 30, 2012.

Conflicts of Interest: The authors report no conflicts of interest. 
$\mathrm{p}=0.03$ ). Patients who required chemotherapy for recurrence after IFRT often benefitted from longer chemotherapy-free intervals after than before IFRT.

Conclusions-Definitive IFRT can yield excellent local control, protracted disease-free intervals, and even cures in carefully selected patients. RT should be considered a tool in the curative management of locoregionally-recurrent ovarian cancer.

\section{Keywords}

Ovarian cancer; radiation therapy; clear cell carcinoma

\section{INTRODUCTION}

Most patients with ovarian cancer present with advanced disease and are treated with surgical resection followed by chemotherapy [1-3]. In the 1960s and 1970s, regional radiation therapy (RT) was used to reduce locoregional progression for early-stage ovarian cancer patients $[2,4-7]$. In the 1970s and 1980s, whole-abdominal irradiation emerged as a useful treatment for selected patients with small-volume disease. However, as more effective systemic treatments were developed, RT was relegated to a palliative role in the United States, rarely considered for definitive treatment $[2,3,7]$.

Up to $70 \%$ of patients with advanced ovarian cancer experience recurrence-usually peritoneal carcinomatosis, although some patients experience predominantly locoregional recurrence without carcinomatosis or distant metastasis [2,3]. After multidisciplinary discussion, we have increasingly treated these patients with local RT, often after repeated localized recurrences despite multiple courses of chemotherapy. We retrospectively reviewed outcomes of such patients to determine the efficacy of definitive involved-field RT (IFRT) in the management of locoregionally-confined recurrent or persistent ovarian cancer.

\section{PATIENTS AND METHODS}

\section{Patients}

We identified 315 women with histologically-confirmed epithelial ovarian cancer treated consecutively with RT at MD Anderson Cancer Center during the period from January 1999 and December 2009. Patients receiving less than $39 \mathrm{~Gy}(\mathrm{n}=210)$ were considered to have received palliative treatment and were excluded. Three patients received 39 to $44 \mathrm{~Gy}$, and were excluded because they were treated with palliative intent. The remaining 102 women, treated with a definitive RT dose of $\geq 45 \mathrm{~Gy}$, were included in this study.

Patient, tumor, treatment, and follow-up information was abstracted from the medical records after Institutional Review Board approval. Ovarian cancer was staged according to the 2009 International Federation of Gynecology and Obstetrics system [8]. Disease sites were classified as pelvic, abdominal, or distant and as nodal or extranodal sites. Distant metastasis included liver metastasis and extra-abdominal disease beyond the paraaortic lymph nodes. 


\section{Treatment}

All patients underwent surgical resection of the primary tumor, bilateral salpingooophorectomy, total abdominal hysterectomy, and selective biopsies and resections of lymph nodes, omentum, and other suspicious findings. One patient received preoperative chemotherapy for liver metastases present at diagnosis. Three high-risk patients received preoperative chemotherapy to allow time for resolution of deep vein thromboses $(n=2)$ and hydronephrosis with acute renal failure $(\mathrm{n}=1)$ before surgery. Surgery was followed by adjuvant platinum-based chemotherapy $(n=96)$ or another chemotherapy regimen $(n=6)$. Seventeen patients underwent second-look laparotomy after adjuvant chemotherapy.

Recurrences before RT were treated with a variety of chemotherapy regimens and/or secondary cytoreduction. Patients were selected to receive definitive IFRT on a case-by-case basis after multidisciplinary discussion. Factors taken into consideration in deciding whether to offer IFRT included the ability to encompass disease by a RT field, timing and pattern of disease progression, length of disease-free intervals, response to prior treatments, resectability of disease, performance status, and limitations of other treatment options, including toxicity. Twenty-seven patients had positron emission tomography (PET) before RT.

Most patients had IFRT for treatment of gross disease ( $\mathrm{n}=73$; median dose $59.2 \mathrm{~Gy}$, range, 45-68.2); however, some patients were treated after gross total resection $(n=16$; median dose 54.5 Gy, range, 45-64.2) or for consolidation following complete response to chemotherapy ( $\mathrm{n}=13$; median dose $48 \mathrm{~Gy}$, range, 45-57.6). Five patients received RT immediately following initial surgery - three for gross residual disease after surgery, one for concurrent endometrial cancer, and one for adenosquamous histology. Twenty patients were treated with high-dose chemotherapy and bone marrow transplant (BMT) for recurrence on an institutional protocol. Of these, 12 patients received IFRT for consolidation after complete response following BMT; the remaining eight had residual or recurrent disease after BMT treated with definitive IFRT ( $\mathrm{n}=5$ ), or resection followed by postoperative RT $(n=3)$.

IFRT was directed to gross disease plus a high-risk clinical target volume (CTV) that included the postoperative bed or the prechemotherapy extent of disease with a $1-$ to $1.5-\mathrm{cm}$ margin, excluding uninvolved clinical structures. Additional CTVs were designated according to the risks of microscopic disease spread, proximity to critical structures, and other risk factors for complications. Nodal CTVs included grossly involved lymph node sites, extending to cover adjacent uninvolved regions. For example, CTVs for patients with paraaortic node recurrence included the entire paraaortic nodal chain, and CTVs for patients with pelvic node recurrence included unilateral or bilateral pelvic nodes. Patients were treated using intensity-modulated RT ( $\mathrm{n}=63)$, 3-dimensional conformal RT ( $\mathrm{n}=35)$, appositional electrons $(n=3)$, or proton beam RT $(n=1)$. Four patients received both external beam RT and vaginal cuff brachytherapy. Eighteen Patients received at least one cycle of concurrent systemic chemotherapy with RT; 14 with platinum-based chemotherapy, three with taxol and one with capecitabine.

Gynecol Oncol. Author manuscript; available in PMC 2015 January 27. 


\section{Statistical Analysis}

Overall survival (OS), progression-free survival (PFS), and in-field disease control were evaluated using Kaplan-Meier estimates and compared using the log-rank test. Clinical and patient factors were compared using Pearson's chi-squared, Mann-Whitney-Wilcoxon, or Wilcoxon ranked-sign tests as appropriate. Ninety-five percent confidence intervals (CI) are reported. Statistical analysis was performed using the Stata software package (StataCorp. 2011. Stata Statistical Software: Release 12. College Station, TX).

\section{RESULTS}

Patient, tumor, and treatment characteristics are shown in Table 1. The median follow-up time after IFRT was 37 months (range, 1-123), and the median follow-up time after diagnosis was 88 months (range, $8-368$ ). The median time from diagnosis to definitive IFRT was 36 months (range, 1-311). Before IFRT, patients received a median of three chemotherapy courses (range, $0-9$ ), and 51 patients had two or more ovarian cancer-related operations (range, $2-4)$. A variety of nodal $(n=50)$ and extranodal $(n=52)$ sites were treated with IFRT.

\section{Survival and Disease Progression}

Five years after IFRT, OS and PFS rates were 40\% (CI=29-50\%) and 24\% (CI=16-33\%), respectively (Fig. 1). Thirty-five patients had no evidence of disease at last follow-up at a median of 38 months after IFRT (range, 7-122). These included 25 patients who remained continuously without disease for a median of 61 months (range, 17-122; Table 2). Patients with clear cell histology were more likely to be continuously without disease after RT ( 6 of $8 ; 75 \%$ ) than other patients ( 19 of $74 ; 20 \% ; \mathrm{p}=0.003$ ). Patients without gross disease at RT were also more likely to be continuously without disease (11 of $29 ; 38 \%$ ) than patients with gross disease ( 14 of $72 ; 19 \%, p=0.05$ ). Ten patients had recurrences at a median of 4.5 months after IFRT (range, 3-39) that were salvaged successfully. These 10 patients remained disease free at a median of 39 months (range, 7-92) after salvage treatment. Three of these 10 patients had isolated nodal recurrences treated with RT alone, resection with postoperative RT, or resection with adjuvant chemotherapy; at last follow-up, these patients were disease free 92, 51, and 7 months, respectively, after salvage treatment. The remaining seven patients had multiple sites of locoregional and distant recurrence treated with combinations of RT $(n=6)$, chemotherapy $(n=4)$, and surgery $(n=3)$.

Eight patients with clear cell carcinoma had higher 5-year OS rates than patients with other histologies $(88 \%, \mathrm{CI}=37-98 \%$, versus $37 \%, \mathrm{CI}=26-47 \%$; $\mathrm{p}=0.05)$ (Fig. 2) as well as higher 5 -year PFS rates $(75 \% \mathrm{CI}=31-93 \%$, versus $20 \%, \mathrm{CI}=12-29 \%$; $\mathrm{p}=0.01)$. Of these eight patients, two had stage I disease at diagnosis, two had stage II, two had stage III and two were unknown. Four clear cell carcinoma patients received IFRT for paraaortic lymph node recurrence, two for extranodal pelvic masses, one to the vaginal cuff and one for an abdominal wall recurrence. Five were treated with definitive IFRT while three had resections prior to RT. Three patients had clear cell carcinoma of the ovary arising in endometriosis. Patients whose disease was sensitive to initial platinum-based chemotherapy, defined as no relapse within 6 months of completion of initial platinum-based

Gynecol Oncol. Author manuscript; available in PMC 2015 January 27. 
chemotherapy, had a higher 5-year OS rate after RT than patients with platinum-resistant disease $(43 \%, \mathrm{CI}=30-54 \%$ versus $27 \%, \mathrm{CI}=8-50 \%, \mathrm{p}=0.03$ ) (Fig. 3), but PFS rates were not significantly different for these two groups. OS and PFS were not statistically significantly different for patients treated with IFRT for gross disease $(38 \%, \mathrm{CI}=26-49 \% ; 18 \%, \mathrm{CI}=10$ $28 \%$ ), after complete resection $(43 \%, \mathrm{CI}=9-74 \% ; 38 \%, \mathrm{CI}=15-60 \%)$, or after complete response to chemotherapy $(39 \% \mathrm{CI}=14-63 \% ; 38 \%, \mathrm{CI}=14-63 \%)$. OS and PFS were not associated with other histologies, stage, grade, time from initial treatment to IFRT, time to first recurrence, number of disease sites at diagnosis, presence of gross disease at IFRT, or age at diagnosis.

\section{In-field Disease Control}

The 5-year rate of in-field disease control after IFRT was 71\% (CI=60-79\%) (Fig. 1). Twenty-seven patients experienced in-field recurrence at a median of 5 months after IFRT (range, 1-35). There was a trend toward higher in-field control rates for tumors $<3 \mathrm{~cm}(76 \%$, $\mathrm{CI}=57-87 \%)$ than for larger tumors $(55 \%, \mathrm{CI}=34-72 \%, \mathrm{p}=0.09)$. The in-field control rate was not statistically significantly different for patients who had IFRT after complete resection $(88 \%, \mathrm{CI}=59-97 \%)$, for patients treated with IFRT for gross disease $(68 \%, \mathrm{CI}=54-$ $78 \%)$, or after complete response to chemotherapy $(68 \%, \mathrm{CI}=35-87 \%)$. In-field control was not associated with histology, site treated with IFRT, presence of gross disease at RT, or RT dose when the presence of gross disease was controlled for.

\section{Regional Disease Control}

Twenty-five patients experienced regional recurrences after IFRT in adjacent, untreated lymph nodes $(n=24)$ or as a focal mass near the previously treated site $(n=1)$. Only two patients experienced isolated regional recurrences without distant metastasis or peritoneal dissemination, and both were successfully salvaged. One patient experienced pelvic node recurrence after IFRT for a vaginal cuff mass invading the bladder and sigmoid colon. She remained disease free 51 months after salvage surgery with postoperative RT. The other patient experienced paraaortic and subdiaphragmatic node recurrence after IFRT for a pelvic sidewall mass and was disease free 7 months after salvage surgery.

\section{Chemotherapy Treatment Breaks}

We hypothesized that IFRT might benefit patients who recur after IFRT through longer breaks from chemotherapy. In patients receiving IFRT for gross disease, we evaluated the time between chemotherapy courses and found that chemotherapy breaks grew progressively shorter with each additional course before IFRT (Fig. 4). We then found that the treatment break immediately before IFRT was significantly shorter (median, 2 months; range, $0-75$ ) than the treatment break immediately after IFRT (median, 6 months; range, 0 $33 ; \mathrm{p}=0.007)$.

\section{Complications}

IFRT was well-tolerated, usually without major acute side effects, although one patient required admission for diarrhea and dehydration near completion of RT. Ten patients had bowel obstructions after RT. One of these patients never received RT to the abdomen or 
pelvis, while the other nine received IFRT to abdominal or pelvic sites. Bowel obstructions for six of the remaining nine patients were associated with active tumor progression. Ultimately, three patients had bowel obstructions without clear evidence of tumor progression. Five of the 10 patients with bowel obstructions had multiple ovarian cancerrelated surgeries before RT (range, 2-5). Five patients had ureteral strictures after RT, all associated with active tumor progression.

Five patients developed second primary neoplasms. Two were diagnosed with acute leukemia; both following BMT and a total of 5 and 9 courses of chemotherapy. One of these two patients died of ovarian cancer. The other was ovarian cancer free 25 months after salvage RT and surgery; however, she ultimately died of acute myeloid leukemia. Two patients developed myelodysplastic syndrome; neither received BMT, but they had 22 and 25 total cycles of chemotherapy. One died of ovarian cancer, and the other was disease free 52 months after salvage surgery, RT, and chemotherapy. One patient developed a squamous cell carcinoma of the oral tongue.

\section{DISCUSSION}

Our findings suggest that definitive IFRT can play an important role in curative management of locoregionally-confined recurrent or persistent ovarian cancer. This is the largest series of patients treated with definitive IFRT for recurrent ovarian cancer reported to date. Over onethird of patients were disease free at last follow-up, and one-fourth were continuously disease free after completion of IFRT at a median of over 5 years of follow-up. While our results indicate that some patients with recurrent ovarian cancer can be cured by RT, the challenge is determining which patients will benefit most from definitive IFRT.

Survival outcomes after IFRT in our study are encouraging in this cohort of patients with multiple recurrences treated with chemotherapy and surgery over a median of three years before even receiving IFRT. Our results compare favorably with results from other smaller series of recurrent ovarian cancer treated with IFRT [9-14] or other treatments $[2,15]$. This indicates that our multidisciplinary selection strategy to identify patients likely to benefit from IFRT was effective.

Our findings with respect to clear cell carcinoma are interesting. Ovarian clear cell carcinoma is typically associated with early-stage disease at presentation, resistance to chemotherapy, and poorer outcomes [16,17]. However, in our series clear cell carcinoma patients had significantly higher OS and PFS rates than other patients. Another smaller series recently reported similar findings [14]. These results indicate that IFRT should be strongly considered for patients with locoregionally-confined recurrences of clear cell carcinoma. However, non-clear cell histology should not preclude receipt of IFRT since prolonged disease-free intervals were observed for these patients as well. In fact, of 13 patients disease free for at least 5 years after IFRT, 11 had serous or mixed histologies and two had clear cell carcinoma.

Platinum sensitivity was also associated with outcomes in this study with platinum-sensitive patients having longer OS after IFRT than platinum-resistant patients. Parallel findings were 
also reported in two other series [12,14]. While platinum-resistant patients had worse outcomes, they still benefitted from IFRT with four of 15 platinum-resistant patients disease free at last follow-up (range, 42 to 117 months).

In-field control in this study also compared favorably to other series [9,11-14]. We observed a trend towards higher in-field control rates for smaller tumors, consistent with other studies $[10,12]$. Another factor hypothesized to result in better local control is the location of recurrent disease. Fujiwara et al. reported higher OS and response rates for nodal than extranodal sites [10]. In this series, we did not observe an association between disease site and outcomes. This was likely due to the variety of sites treated and small numbers on subset analysis. We suspect that site may still be an important prognostic factor, and should be studied further.

Secondary cytoreductive surgery for recurrent disease with or without additional chemotherapy may improve outcomes, especially for patients with optimal cytoreduction, prolonged disease-free intervals before resection, and a single site of recurrence [15,18-21]. RT may be an excellent alternative to resection. In our study, the presence or absence of gross disease was not significantly associated with survival or local control. Furthermore, outcomes in our study and other IFRT series compare favorably with outcomes in studies of secondary cytoreductive surgery for limited ovarian cancer recurrence [15,18-21]. It is unclear if gross total resection of recurrent disease before IFRT improves local control. Albuquerque et al. reported higher 3-year local-recurrence-free survival rates following optimal resection (89\%) than for patients with gross disease (42\%) [9]. In our study in-field control rates were not significantly different for patients with completely resected recurrent disease. A multidisciplinary discuss should consider how best to use surgery and RT to treat localized recurrences. Surgery may be useful for patients with large-volume disease as long as complete resection can be achieved. However, definitive RT should be strongly considered if complete resection is unlikely since anything less than complete resection would likely not improve local control and might increase complications with postoperative RT. It is unclear whether additional chemotherapy should be given following resection or IFRT for localized recurrences; this should be studied further.

The potential for complications should also be taken into consideration when making treatment recommendations for patients with locoregionally-recurrent ovarian cancer [18,21]. RT can contribute to complications, including bowel obstruction, diarrhea, and ureteral strictures. However, the etiology of many complications is most likely multifactorial, given the multiple surgeries, several courses of chemotherapy, and tumor progression that these patients endured. Chemotherapy likely contributed to subsequent primary leukemias and myelodysplastic syndromes observed in this study. Chemotherapy may have also contributed to the oral tongue cancer developed in a lifetime never smoker after receiving 13 cycles of doxorubicin, which has been associated with oral cavity malignancies in nonsmoking women treated with pegylated liposomal doxorubicin for ovarian cancer [22]. Multidisciplinary discussions should include an evaluation of potential toxicities for each available treatment option. Overall, RT in this study was well-tolerated without significant side effects. 
Several unanswered questions remain. First, might some patients benefit from earlier RT? In this study, patients did not receive IFRT until a median of 3 years after diagnosis, after a median of three chemotherapy courses. One patient with limited disease had eight chemotherapy courses and two resections before ultimately being rendered disease free by IFRT. For patients like this, earlier IFRT might improve disease outcomes and reduce additive toxicity of multiple courses of chemotherapy. Further study is warranted to identify clinical and molecular factors associated with locoregionally-confined recurrence so that local treatment can be administered early in the progression of the disease. We also suggest PET to confirm the absence of distant disease spread before recommending RT.

A second unanswered question is, are the IFRT target volumes we used appropriate, or should they be expanded to prevent regional recurrences? Field design was fairly subjective, involving clinical judgments about risks of microscopic disease spread and the potential for complications. In this study, only two of 25 regional recurrences occurred in the absence of simultaneous distant or peritoneal metastasis; both successfully salvaged. Based on these results, we treat gross tumor with a CTV expansion to involved nodal levels without extending fields to other adjacent regions. This approach balances the need to deliver RT doses sufficient to eradicate gross disease with the goal to minimize adverse effects from larger fields. Patients with regional recurrences without further dissemination can be successfully salvaged with additional IFRT or surgery. We also recommend against large doses per fraction using stereotactic ablative body radiation because of the risk for toxicity when target volumes are near sensitive clinical structures as well as because the small fields required to treat with large doses per fraction may not fully encompass microscopic disease spread, potentially resulting in higher rates of locoregional failure. Further study is needed to determine the most appropriate RT techniques, volumes, and doses for this patient population.

This study has several limitations, including those inherent in retrospective studies. Many patients were selected for IFRT because they exhibited a favorable pattern of recurrence. Unfortunately, this population of patients with locoregionally-confined recurrence has not been well described in the literature, making it difficult to compare the efficacy of IFRT versus other treatments. The selection process yielded a diverse group of patients with respect to tumor sites, histologies, and prior treatments, which made comparisons possible but also resulted in small subset sizes.

Despite these limitations, this study suggests that definitive IFRT can yield excellent local control, protracted disease-free intervals, and cures in carefully selected patients. Even patients who progress following IFRT may benefit from longer chemotherapy breaks after RT. It was difficult to identify specific findings associated with good outcomes after IFRT because of the variety of patient presentations in this study. We believe that IFRT may be particularly useful for patients with a good response to prior treatment, long intervals without disease progression and for localized sites of recurrence in patients without carcinomatosis or distant metastasis. Patients with clear cell histology may be particularly good candidate for definitive treatment. However, many patients without these factors also had good outcomes following IFRT. As such, we recommend that RT should be considered as a tool in the curative management of all patients with locoregionally-confined recurrent or 
persistent ovarian cancer. Since most patients in this study received IFRT after multiple courses of chemotherapy, we also recommend further study to determine if earlier initiation of IFRT can reduce additive chemotherapy toxicity. Future work should focus on identifying clinical and molecular characteristics of patients most likely to benefit from definitive IFRT, as well as optimizing radiation doses, volumes, and techniques.

\section{REFERENCES}

1. Siegel R, Naishadham D, Jemal A. Cancer statistics, 2012. Cancer. 2012; 62:10-29.

2. Herzog TJ, Pothuri B. Ovarian cancer: a focus on management of recurrent disease. Nature Clin Pract Oncol. 2006; 3:604-611. [PubMed: 17080178]

3. Cannistra SA. Cancer of the ovary. New Engl J Med. 2004; 351:2519-2529. [PubMed: 15590954]

4. Thomas GM, Dembo AJ. Integrating radiation therapy into the management of ovarian cancer. Cancer. 1993; 71:1710-1718. [PubMed: 8431909]

5. Thomas GM. Radiotherapy in early ovarian cancer. Gynecol Oncol. 1994; 55:1541-1546.

6. Fyles A, Bolis G, Ferraris C, Bolla M, Parazzini F. Is abdomino-pelvic radiation therapy the optimal treatment for completely resected stage I and II high risk ovarian cancer? Eur J Cancer. 1997; 33:12-19. [PubMed: 9071893]

7. Cardenes H, Randall ME. Integrating radiation therapy in the curative management of ovarian cancer: current issues and future directions. Semin Radiat Oncol. 2000; 10:61-70. [PubMed: 10671660]

8. Current FIGO staging for cancer of the vagina, fallopian tube, ovary, and gestational trophoblastic neoplasia. Int J Gynecol Obstet. 2009; 105:3-4.

9. Albuquerque KV, Singla R, Potkul RK, Smith DM, Creech S, Lo S, et al. Impact of tumor volumedirected involved field radiation therapy integrated in the management of recurrent ovarian cancer. Gynecol Oncol. 2005; 96:701-704. [PubMed: 15721414]

10. Fujiwara K, Suzuki S, Yoden E, Ishikawa H, Imajo Y, Kohno I. Local radiation therapy for localized relapsed or refractory ovarian cancer patients with or without symptoms after chemotherapy. Int J Gynecol Cancer. 2002; 12:250-256. [PubMed: 12060445]

11. Davidson S, Rubin S, Mychalczak B, Saigo PE, Lewis JL, Chapman D, et al. Limited-field radiotherapy as salvage treatment of localized persistent or recurrent epithelial ovarian cancer. Gynecol Oncol. 1993; 51:349-354. [PubMed: 8112644]

12. Lee SW, Park SM, Kim YM, Kim YS, Choi EK, Kim DY, et al. Radiation therapy is a treatment to be considered for recurrent epithelial ovarian cancer after chemotherapy. Tumori. 2011; 97:590 595. [PubMed: 22158489]

13. Yahara K, Ohguri T, Imada H, Moon S, Yamaguchi S, Narisada H, et al. Epithelial Ovarian Cancer: Definitive Radiotherapy For Limited Recurrence After Complete Remission [Abstract]. ASTRO Annual Meeting. 2011

14. Lee LJ, Newhouse C, Chen Y, Viswanathan A. Nonserous Histology and Platinum-sensitivity are Prognostic in Localized Recurrent Ovarian Cancer Treated With Radiation Therapy [Abstract]. ASTRO Annual Meeting. 2012

15. Bristow RE, Puri I, Chi DS. Cytoreductive surgery for recurrent ovarian cancer: a meta-analysis. Gynecol Oncol. 2009; 112:265-274. [PubMed: 18937969]

16. Lee YY, Kim TJ, Kim MJ, Kim HJ, Song T, Kim MK, et al. Prognosis of ovarian clear cell carcinoma compared to other histological subtypes: a meta-analysis. Gynecol Oncol. 2011; 122:541-547. [PubMed: 21640372]

17. del Carmen MG, Birrer M, Schorge JO. Clear cell carcinoma of the ovary: a review of the literature. Gynecol Oncol. 2012; 126:481-490. [PubMed: 22525820]

18. Salani R, Santillan A, Zahurak ML, Giuntoli RL, Gardner GJ, Armstrong DK, et al. Secondary cytoreductive surgery for localized, recurrent epithelial ovarian cancer: analysis of prognostic factors and survival outcome. Cancer. 2007; 15(109):685-691. [PubMed: 17219441] 
19. Chi DS, McCaughty K, Diaz JP, Huh J, Schwabenbauer S, Hummer AJ, et al. Guidelines and selection criteria for secondary cytoreductive surgery in patients with recurrent, platinum-sensitive epithelial ovarian carcinoma. Cancer. 2006; 1(106):1933-1939. [PubMed: 16572412]

20. Zang RY, Li ZT, Tang J, Cheng X, Cai SM, Zhang ZY, et al. Secondary cytoreductive surgery for patients with relapsed epithelial ovarian carcinoma: who benefits? Cancer. 2004; 100:1152-1161. [PubMed: 15022281]

21. Tebes SJ, Sayer RA, Palmer JM, Tebes CC, Martino MA, Hoffman MS. Cytoreductive surgery for patients with recurrent epithelial ovarian carcinoma. Gynecol Oncol. 2007; 106:482-487. [PubMed: 17590420]

22. Cannon TL, Lai DW, Hirsch D, Delacure M, Downey A, Kerr AR, et al. Squamous Cell Carcinoma of the Oral Cavity in Nonsmoking Women: A New and Unusual Complication of Chemotherapy for Recurrent Ovarian Cancer? Oncologist. 2012; 17 


\section{HIGHLIGHTS}

- Selected ovarian cancer patients with locoregionally-confined recurrences were treated with definitive involved-field radiation therapy (IFRT).

- IFRT produced high rates of in-field disease control and prolonged disease-free intervals of up to 10 years in some patients.

- Some patients with disease progression following IFRT may benefit from RT through longer breaks from chemotherapy. 


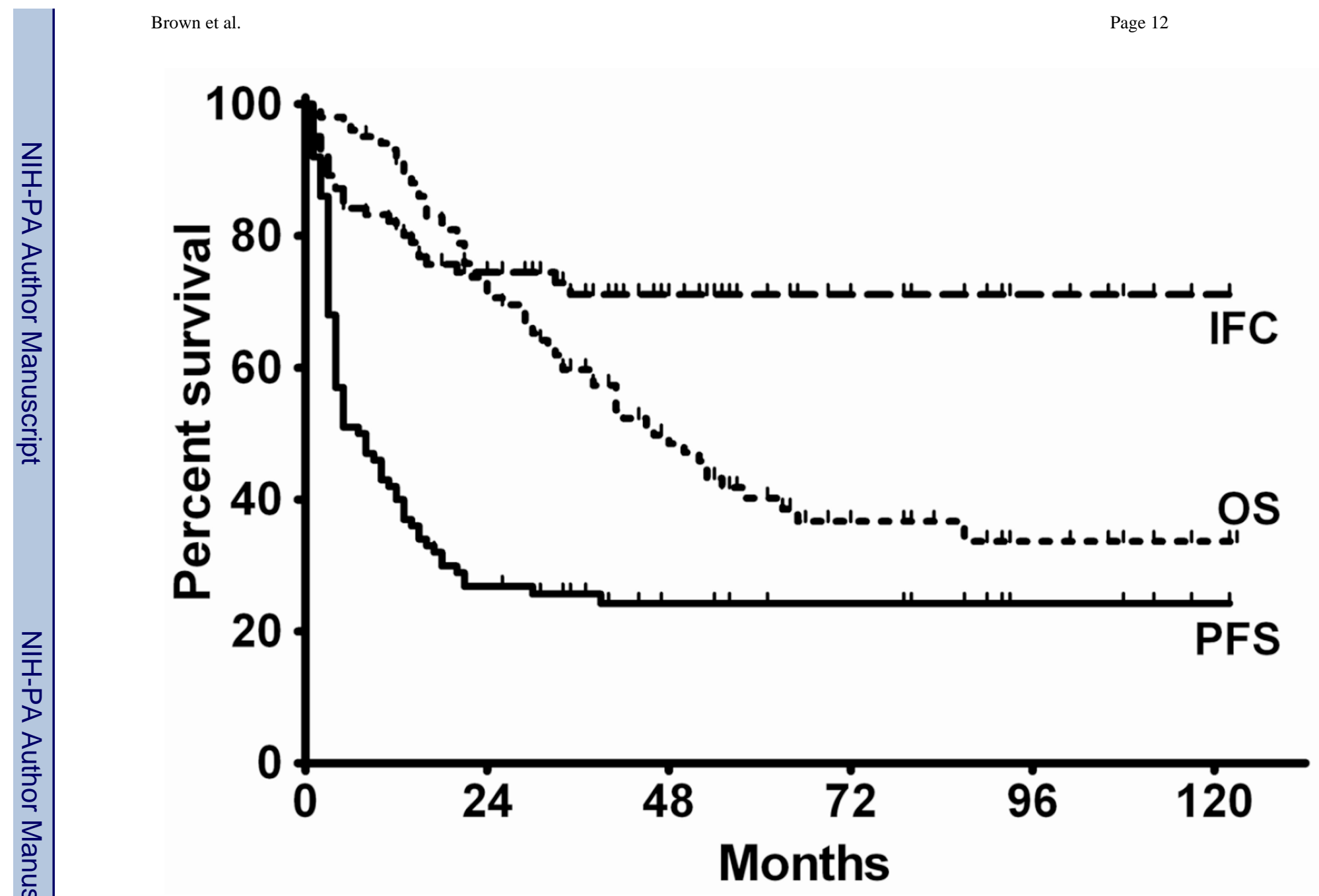

Figure 1.

Kaplan-Meier curves showing overall survival (OS), progression-free survival (PFS), and in-field control (IFC). 


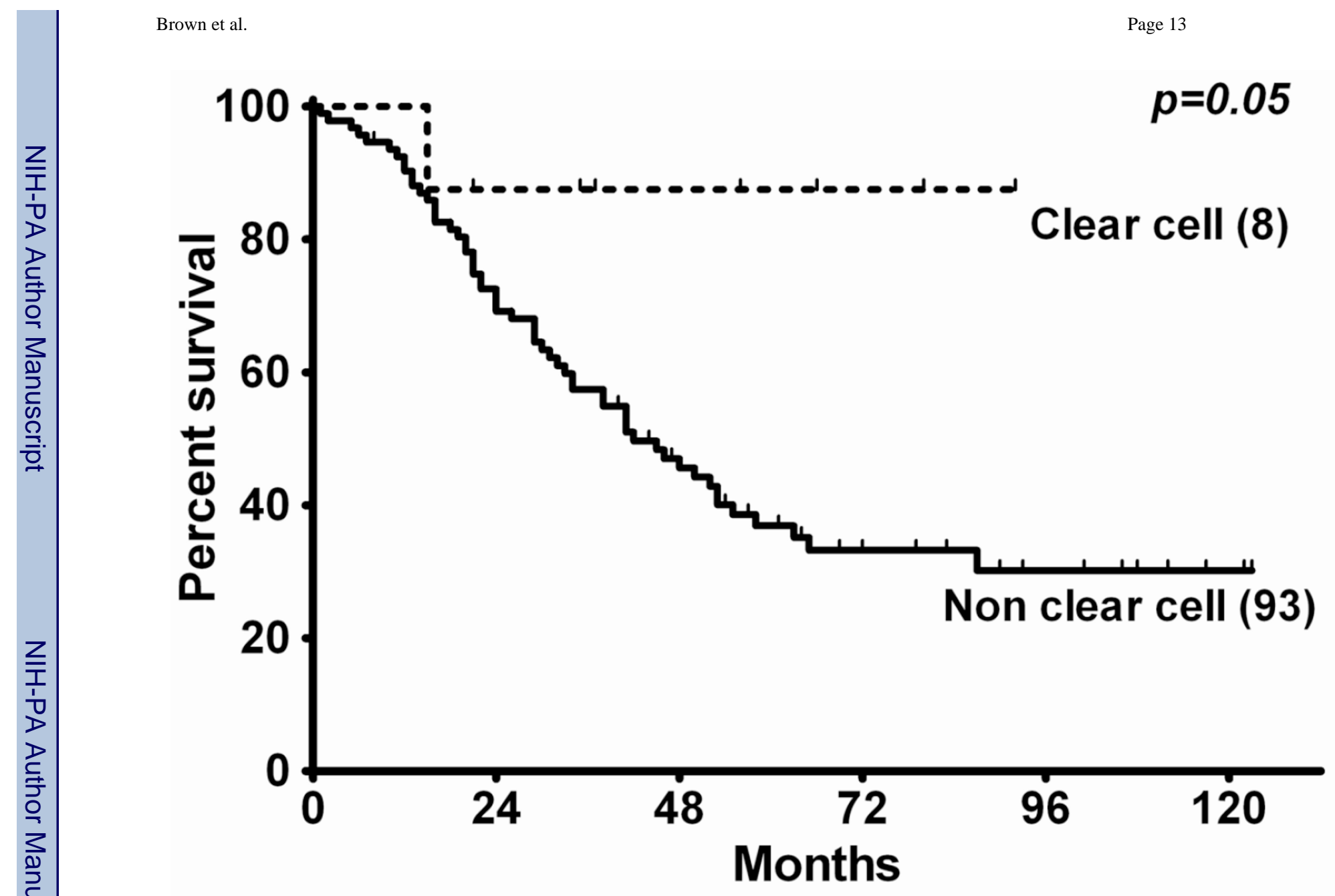

Figure 2.

Patients with clear cell carcinoma had significantly better overall survival than patients with other histologies. 


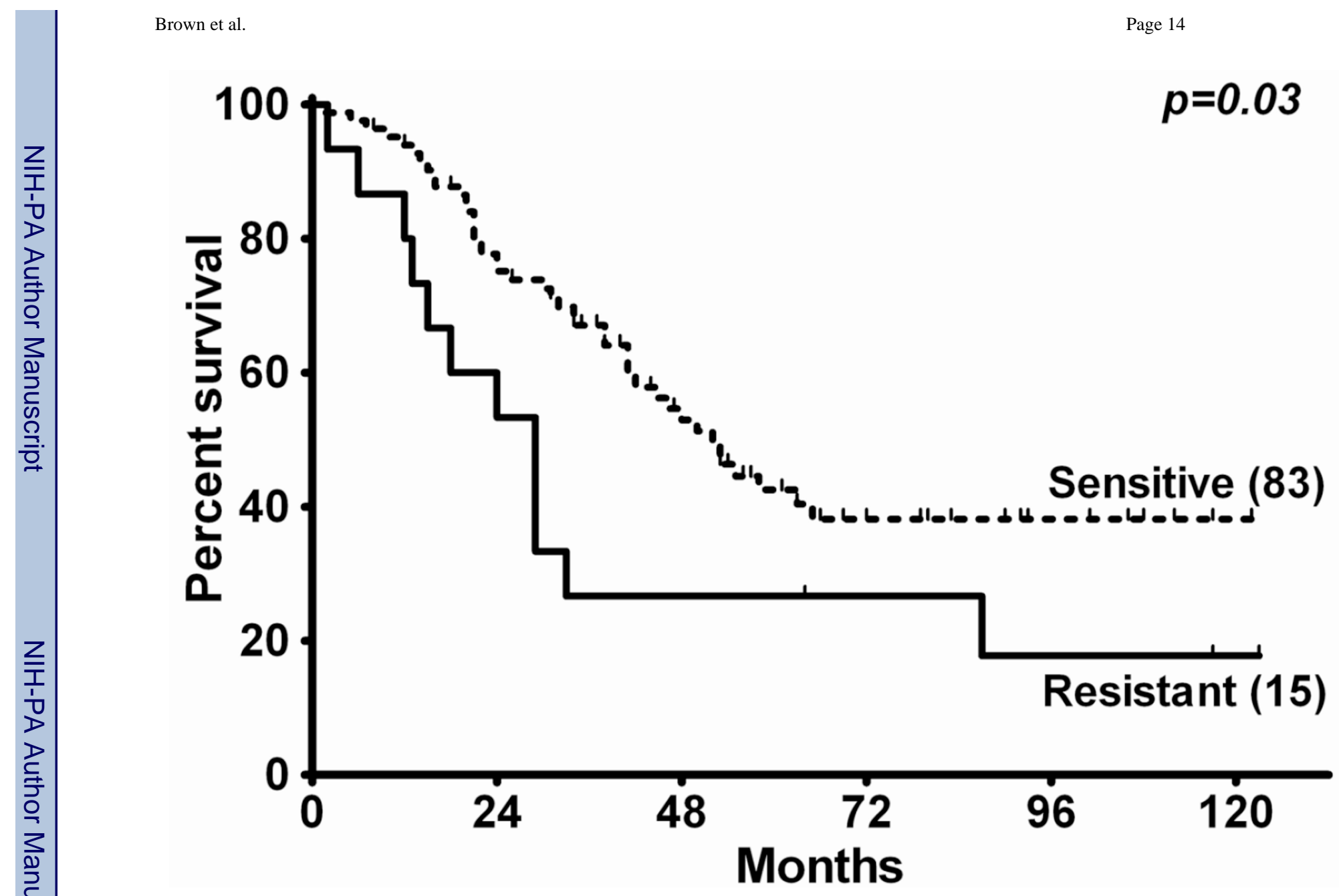

Figure 3.

Patients whose disease was sensitive to the initial course of platinum-based chemotherapy had significantly better overall survival than patients whose disease was platinum resistant. 


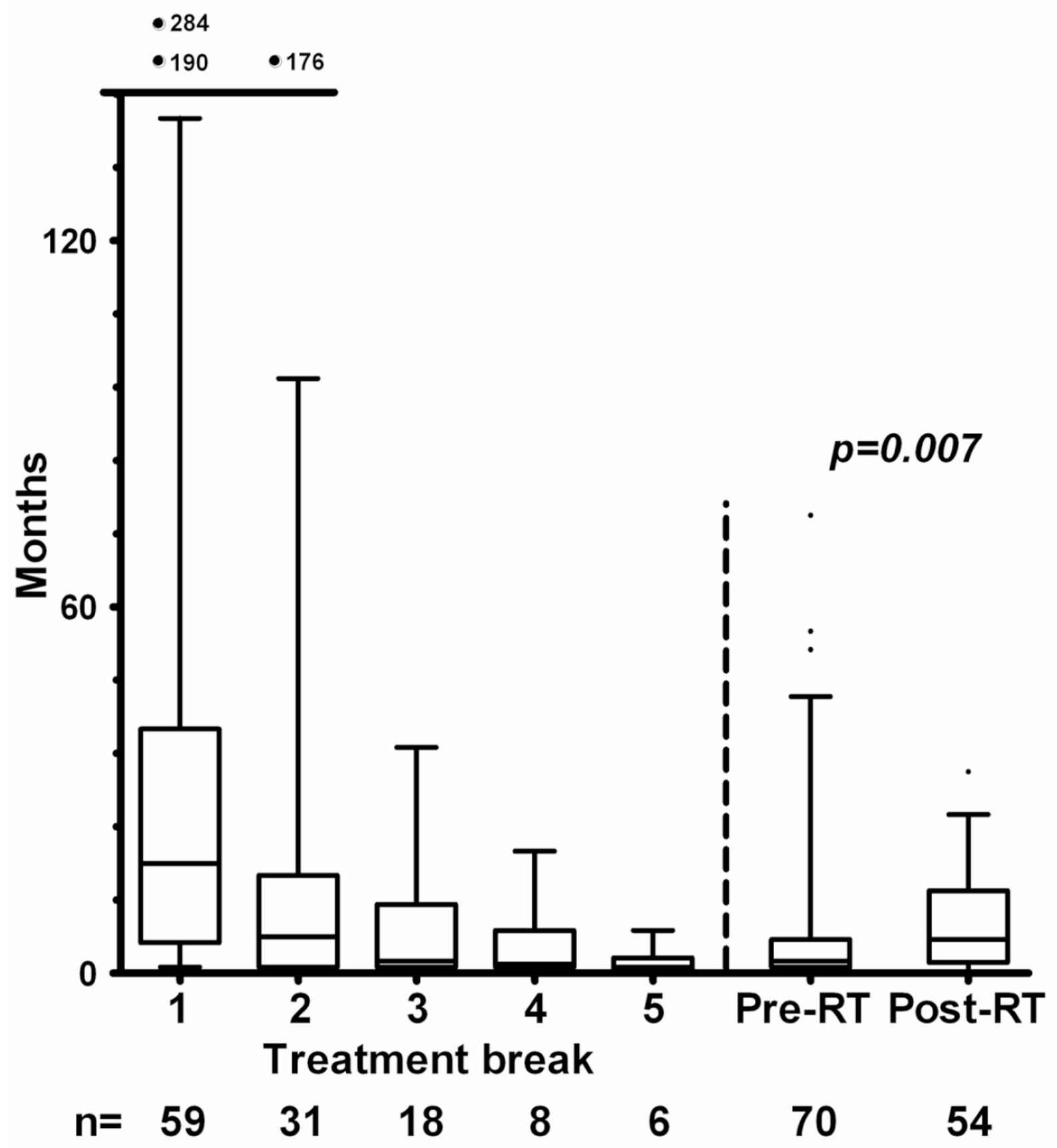

Figure 4.

Box and whisker plot (range, 5th-95th percentile) showing progressively shorter treatment breaks between courses of chemotherapy for each additional course before radiation therapy (RT) in patients with gross disease. The median treatment break immediately after RT (6 months) was significantly longer than the median treatment break immediately before RT ( 2 months). 


\section{Table 1}

Patient, tumor, and treatment characteristics $(\mathrm{N}=102)$.

\begin{tabular}{|c|c|}
\hline Characteristic & \\
\hline Age at diagnosis, years, median (range) & $53(29-78)$ \\
\hline Age at RT, years, median (range) & $58(30-81)$ \\
\hline Time to first recurrence, months, median (range) & $19(0-288)$ \\
\hline Time to RT, months, median (range) & $36(1-311)$ \\
\hline \multicolumn{2}{|l|}{ Histology, no (\%) } \\
\hline Serous & $55(54)$ \\
\hline Mixed & $17(17)$ \\
\hline Endometrioid & $10(10)$ \\
\hline Clear cell & $8(8)$ \\
\hline Mucinous & $5(5)$ \\
\hline Other & $7(7)$ \\
\hline \multicolumn{2}{|l|}{ Stage, no (\%) } \\
\hline I & $15(15)$ \\
\hline II & $18(18)$ \\
\hline III & $58(57)$ \\
\hline IV & $4(4)$ \\
\hline Unknown & $7(7)$ \\
\hline \multicolumn{2}{|l|}{ Grade, no (\%) } \\
\hline 1 & $5(5)$ \\
\hline 2 & $8(8)$ \\
\hline 3 & $84(82)$ \\
\hline Unknown & $5(5)$ \\
\hline \multicolumn{2}{|l|}{ Sites treated with RT, no $(\%)$} \\
\hline \multicolumn{2}{|l|}{ Pelvis } \\
\hline Pelvic LN & $10(10)$ \\
\hline Vaginal cuff & $21(21)$ \\
\hline Other pelvic mass & $23(23)$ \\
\hline \multicolumn{2}{|l|}{ Abdomen } \\
\hline Paraaortic LN & $27(26)$ \\
\hline Abdominal wall mass & $2(2)$ \\
\hline Other abdominal mass & $6(6)$ \\
\hline Inguinal LN & $10(10)$ \\
\hline Supraclavicular LN & $2(2)$ \\
\hline Mediastinal LN & $1(1)$ \\
\hline \multicolumn{2}{|l|}{ RT indication, no (\%) } \\
\hline Treatment of gross disease & $73(71)$ \\
\hline Postoperative treatment & $16(16)$ \\
\hline Consolidation after chemotherapy & $13(13)$ \\
\hline Endocrine therapy & $41(40)$ \\
\hline
\end{tabular}




\begin{tabular}{ll}
\hline Characteristic \\
\hline Secondary cytoreduction & $46(45)$ \\
\hline
\end{tabular}

$\mathrm{RT}=$ radiation therapy; $\mathrm{LN}=$ lymph node . 


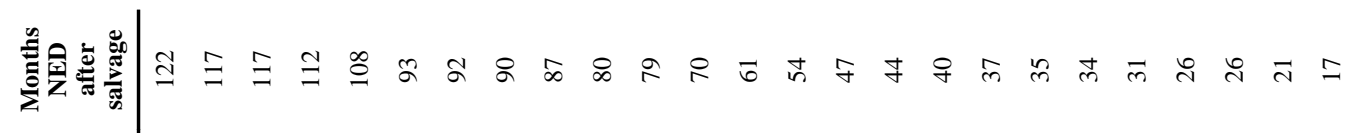

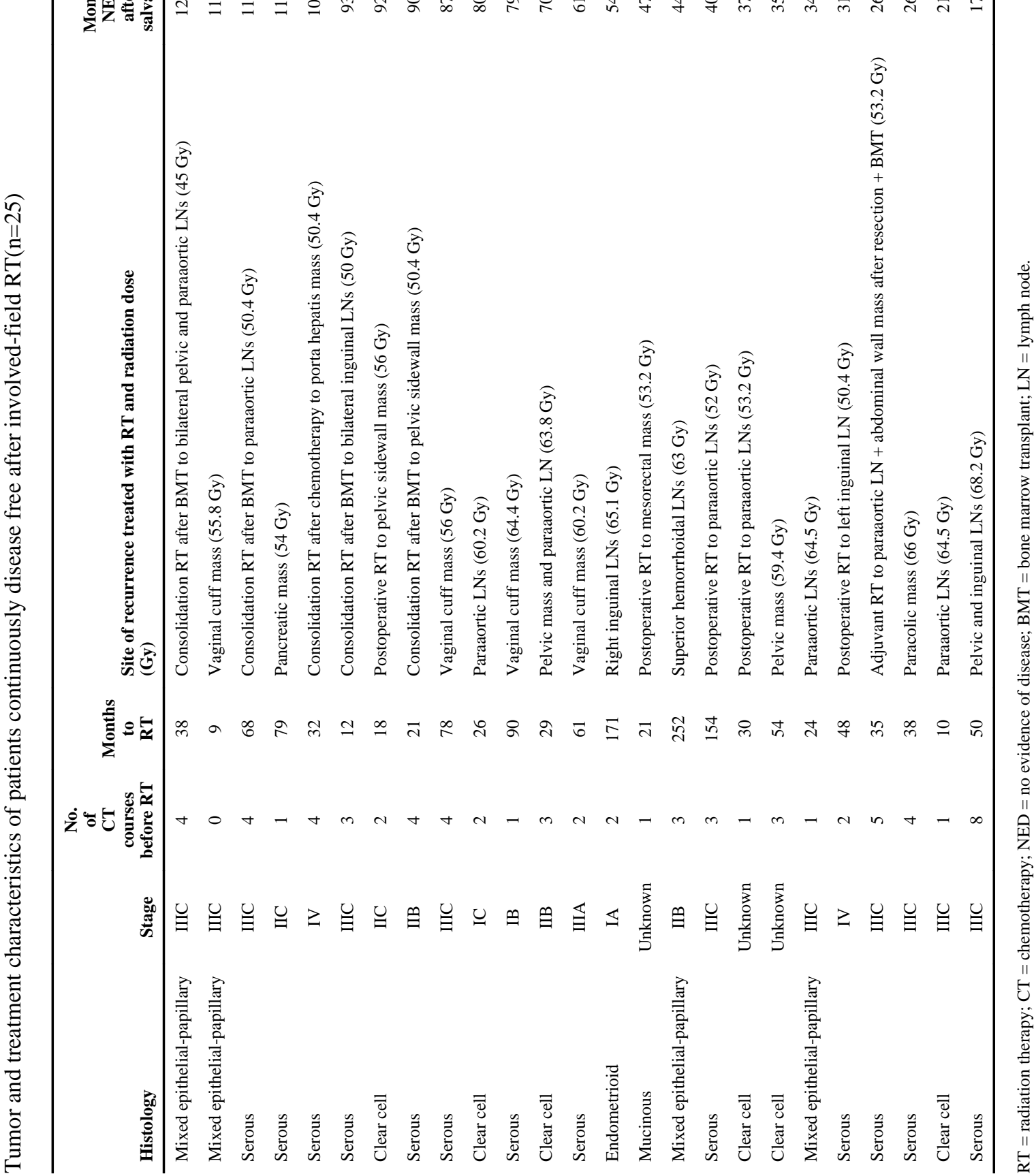

